\title{
Racial differences in HF among young adults
}

In the US, the incidence of heart failure is 20 times higher in black men and women in their 30s and 40s than in white adults in the same age group. Cardiovascular risk factors also develop much earlier in young black adults.

The CARDIA study was conducted to define how early heart failure develops in black and white young adults. The study cohort consisted of 5,115 men and women aged 18-30 years at the time of enrolment in 1985-1986. Heart failure in black men and women under 50 years occurred at a rate of 1 in 100 , which is similar to that seen in white adults in their 50s and 60s. Risk factors, in particular hypertension, obesity and diabetes, were present in black participants when they were in their 20 s and 30 s and heart failure occurred at an average age of 39 years.
The CARDIA study highlights the importance of preventing or treating risk factors, such as hypertension and obesity, in young adults. Kirsten BibbinsDomingo, one of the CARDIA researchers commented that "the consequence of failing to focus on these factors in young adults can be devastating illness by middle age". More work is required to determine why the black population in the US is predisposed to heart failure at a younger age than the white population, and whether genetic or environmental factors contribute to the increased risks.

\section{Sharmini Rajanayagam}

Original article Bibbins-Domingo, K. et al. Racial differences in incident heart failure among young adults. N. Engl. J. Med. 360, 1179-1189 (2009). 